\title{
Facilitating Constructive Alignment in Power Systems Engineering Education Using Free and Open-Source Software
}

\author{
Luigi Vanfretti, Member, IEEE, and Federico Milano, Senior Member, IEEE
}

\begin{abstract}
This paper describes how the use of free and open-source software (FOSS) can facilitate the application of constructive alignment theory in power systems engineering education by enabling the deep learning approach in power system analysis courses. With this aim, this paper describes the authors' approach in using the Power System Analysis Toolbox (PSAT) for undergraduate and graduate education. Interviews with former students reveal the positive impact that the use of FOSS in general, and PSAT in particular, had on their learning and how it has influenced their professional life.
\end{abstract}

Index Terms - Constructive alignment, free and open-source software (FOSS), functioning knowledge, learning activities, power system analysis.

\section{INTRODUCTION}

$\mathbf{T}$ HE POWER and energy industry is facing one of its biggest challenges ever: overcoming the looming shortage of human resources due to the combination of an aging workforce and an insufficient number of newly qualified engineers. Although this phenomenon was first identified as an important issue during the early 2000 s, primarily impacting North America [1], [2], it continues to be a major concern [3]. While earlier reports of this kind of shortage were limited to North America, this phenomenon has now spread to other industrialized societies, including the European Union [4] and Australia [5].

A report issued by the House of Commons of the United Kingdom in 2009 states that " $40 \%$ of National Grid's workforce will reach retirement age over the next $10-15$ years. The UK faces a 'crucial skills shortage from 2015 to 2025 that will make power supplies less reliable and more expensive"” [6]. A press release from Nordic Energy Research in 2008 [7] states

Manuscript received June 19, 2011; accepted September 27, 2011. This work was supported by the Swedish Research Council (Vetenskapsrådet, naturvetenskaplig-teknikvetenskaplig forskning) under Grant B0000601 (Diarinummer: 624-2011-6, Avtals-ID: B0000601). L. Vanfretti was supported by the STandUP for Energy collaboration initiative and the KTH School of Electrical Engineering. F. Milano was supported in part by the Ministry of Science and Education of Spain under CICYT Project ENE-2009-07685 and Junta de Comunidades de Castilla—La Mancha under Project POII11-0148-1022.

L. Vanfretti is with the Electric Power Systems Division, School of Electrical Engineering, KTH Royal Institute of Technology, 10044 Stockholm, Sweden (e-mail: luigiv@kth.se).

F. Milano is with the Department of Electrical Engineering, University of Castilla-La Mancha, 13071 Ciudad Real, Spain (e-mail: Federico.Milano@uclm.es).

Digital Object Identifier 10.1109/TE.2011.2172211 that in Denmark "for every new power engineer who completes his/her education, three power engineers are retiring."

The difficulties of attracting new talent into this particular engineering area are large [8], and replacing these retiring engineers by training new human resources is increasingly challenging. The problem is not simply one of the supply and demand of human resources - there is also the need to appropriately transfer knowledge [7], [8] so that new engineers arrive in the workforce with functioning knowledge.

This paper lays out different educational approaches that try to answer the general questions arising from the challenges outlined above. It does this by focusing on a particular topic: the use of free and open-source software (FOSS) in undergraduate as well as graduate power engineering education as a tool for facilitating the application of constructive alignment theory. This paper builds on the authors' experience in developing and using a specific FOSS, the Power Systems Analysis Toolbox (PSAT) [9] (as described in [10]-[12]), to examine how FOSS can be an enabler of the deep learning approach in power system courses through its use in teaching and learning activities.

There is a strict relationship between enabling deep learning and allowing students to join to the workforce with functioning knowledge. A good teaching approach can stir the curiosity of students with potentially promising skills and make it more likely that deep learning is triggered. This is a key factor if constructive alignment theory is to be implemented efficiently in power systems engineering education.

The authors' main conclusion, after working for almost a decade in power engineering education, is that FOSS is inherently attractive to young people. This is most likely because FOSS is somewhat "outside conventional schemes." This is a notion of "rebellion" that is always appealing for new generations. The important point behind FOSS is that it takes advantage of this notion of generating a positive and productive contribution to society by going outside of the conventional schemes.

PSAT shows students that a free software tool is capable of solving the same problems that costly proprietary software packages solve. Furthermore, PSAT can be opened, and the "mystery" behind the internal functioning of a power system package can be unveiled. Creative students quickly discover through the use of PSAT that power engineering really can be mastered and that it is more accessible and user-friendly than it may seem at first sight.

The second author has recently developed the next generation of PSAT by using the Python language [13] as a tool used 
exclusively for research activities and by senior Ph.D. students. The main goal of this Python project was to facilitate as much as possible the development of new device models and prototypes of novel algorithms. After a couple of years of development and use of this new tool, the main conclusion is that a strict relationship exists between a software architecture and the actual triggering of deep learning. In other words, FOSS per se is not enough to stimulate the participation of students in the development of an open-source project. The project itself has to be properly structured in order to be easily understood. The choice of programming language and software architecture thus plays a critical role.

PSAT sweeps away the paradigm imposed by monolithic proprietary packages that complacently obscure, through unnecessary stratum, theory, and methods that otherwise are intelligible and even elegant. This is beneficial for two reasons. First, students learn that even complex software projects, such as PSAT, can be mastered if properly approached. Second, students understand that any software package can be improved, extended, and customized. The latter skill is more commonly achieved by $\mathrm{Ph}$.D. students, but is also attainable for motivated undergraduate students.

The intellectual skills that students need to develop, which can later be applied to any particular practical problem, are curiosity, analysis and synthesis ability, and intellectual honesty. FOSS (and thus PSAT) are effective in achieving this.

The objectives of this article are the following:

1) to describe the relationship between FOSS in general, and PSAT in particular, and deep learning in undergraduate courses;

2) to describe the relationship between a "good" open-source software architecture and deep learning for Ph.D. research activities;

3) to provide feedback from former students, now practicing engineers, who used PSAT while taking power engineering courses.

The remainder of this article is organized as follows. Section II defines surface and deep learning approaches. Sections III and IV describe the authors' experience with the use of PSAT for undergraduate and graduate learning and for teaching activities, respectively. Section V provides excerpts from interviews with practicing engineers who, as former students, used PSAT in their courses. Finally, Section VI draws conclusions, and Appendix B depicts the interview that was submitted to former students.

\section{LEARNING APPROACHES: SURFACE AND DEEP LEARNING}

The concepts of surface and deep learning approaches arise from studies of the contexts in which learning is pursued, that is to say higher learning institutions; these studies gave birth to the field of student learning research. Although these concepts are not new and have been properly addressed in the past (see, for example, the school reform elaborated by Gentile in Italy at the beginning of the 20th century [14], [15]), the terms surface and deep learning, as used in Constructive Alignment (CA) theory, ${ }^{1}$ originated in Sweden [17], [18] in the 1970s.

\footnotetext{
${ }^{1}$ For details on CA theory, the interested reader is referred to [16].
}

As the term suggests, the surface learning approach occurs when students focus only on covering the superficial layer of a course's content. The term was coined by Marton and Säljö, who noticed two kinds of responses from students after assessing a reading assignment [17], [18]. The response from those using the surface approach was characterized by a strong focus on memorization of facts and details without joining these pieces of information together. Although these students had a recollection of terms and isolated items, they did not show an overall understanding of the underlying ideas conveyed in the reading assignment.

Several factors trigger a surface or deep learning approach in student learning [16], the most important of which is the lack of alignment between the intended learning outcomes (ILOs), the teaching and learning activities (TLAs), and the course assessment and grading. A brief outline some of these aspects is provided here.

\section{A. Surface Learning Approach}

This approach is characterized by a deliberate need to get the course tasks out of the way while giving an appearance of complying with the course requirements - in other words, the "principle of least effort" in action. Memorization is a surface approach used to counterfeit understanding. Selected content is learned by rote, leaving a poorly structured knowledge that is not capable of withstanding the test of time.

Nevertheless, the surface learning approach is not an attitude conceived by the student independently; it is actually the response of the student to the teaching and assessment conditions. As pointed out by Gibbs and Tang [19], it is possible to say that "under current conditions of teaching or assessment, he (the student) chooses to use the surface approach." When the assessment system is not aligned with the learning objectives and the teaching activities, the students are unable to see the structure and significance of what is being taught. Hence, it is possible to satisfy the course requirements, and even to get a good grade [20], [21], if the assessment system rewards rote learning. However, those good examination results will not necessarily guarantee any retention and actual learning.

\section{B. Deep Learning Approach}

In this approach, students attach value and meaning to their learning process. Motivated by a "need to know," they undertake the different course tasks seriously. Because of this commitment, they learn the details associated in the learning tasks, as well as understanding the ideas behind these details. As a consequence, a solid knowledge foundation is established that allows students to also understand the "big picture," thus deriving satisfaction and positive feelings. It is interesting to note that this approach is implicit in the Liceo Classico and university courses described by Gentile in [14]. In such an educational scheme, surface learning will not suffice for passing examinations since mere memorization is far from being enough to attain satisfactory marks. However, the main drawback of Gentile's approach is that students who have no natural disposition for the deep learning approach typically abandon their studies.

Nevertheless, the student factors mentioned above are not independent of teaching strategies, and the deep approach will not 
be triggered if the course ILOs, TLAs, and assessment are not aligned. Proper design of each of these factors will aid the students in adopting a deep approach. It is highlighted in [22] that students are most drawn to the deep approach when "the route to understanding is through application." The remainder of this section discusses how different TLAs can be designed with the aid of FOSS to trigger the deep learning approach.

\section{Remarks on Surface and Deep Learning Approaches}

It is worth observing that one learning approach is not exclusive of the other; a student can use both a deep and surface approach in the same course [22]. While a course project or its assignments can trigger the deep learning approach, the approach taken for an examination will likely be a surface one if no changes to traditional assessment are made. As it appears from [20] and [21], and as it will be shown later, the deep approach results in long-term functioning knowledge and retention. However, the impact of these activities is not fully realized if the entire course assessment is not modified along the lines of CA [16].

\section{TRIgGering DeEP LEARNING IN UndergraduATE COURSES}

This section discusses the design and use of learning activities that, through the utilization of FOSS, trigger the deep learning approach and therefore predispose toward functioning knowledge. These learning activities use important elements of CA theory [16]. CA theory and the recommendations in [22] can be observed in the design of the learning activity and the assessment task as described in what follows.

\section{A. Preparatory Learning Activities}

The project was used both as a learning activity and an assessment task. Preparation for this course project helped students in getting started with their own project. With this in mind, several activities carried out before the course project prepared the students on two fronts: indicating to them which theory they should understand from the course, and building their competence on use of the software tool. These preliminary activities are the following:

1) an information session on using the FOSS PSAT for power flow analysis [23];

2) a learning activity focusing on power flow analysis as described in [10] (see Section V) carried out after the information session;

3) a homework assignment on power flow analysis using "paper and pencil" with optional use of PSAT [24].

Note that TLA 1) focuses in building declarative knowledge, while 2) and 3) provide opportunities for functioning knowledge at the relational level. Functioning knowledge at the extended abstract level [16] was achieved through the project described here.

It is important to highlight that the simple class activity of item 2) is most likely only possible due to the open-source nature of PSAT. Unlike commonly used commercial proprietary software used for power system analysis, FOSS allows the software source code to be changed.

\section{B. Project Design and Elements for Functioning Knowledge That Trigger the Deep Learning Approach}

1) Design and Elements: Teaching is about providing students with the opportunity to learn appropriately, without resorting to shortcuts. This can only be accomplished by encouraging them to adopt the deep learning approach. The course project assigned to the students, outlined in Appendix A, is a case-based learning activity with group work format.

The ILOs of many engineering courses cite the application of knowledge in practical contexts, yet traditional course assignments provide little opportunity for this [16]. The first step is to build a declarative knowledge base (as described in the previous section) that the students can exploit to apply their knowledge and to make informed decisions. The next step is to put this knowledge into action, as in the proposed project.

The project considered a real-life design, with its various constraints and limitations. The students were asked to develop two designs and, by using their techno-economical engineering judgment, to select the design that met the design specifications. The goal of the project is to trigger the deep learning approach at the different cognitive levels of the Structure of Observed Learning Outcome (SOLO) taxonomy [25] while putting the emphasis on the higher cognitive levels. To this aim, the "Suggested Project Tasks and Guidelines" encouraged the students on the three highest levels of the SOLO taxonomy (the active verbs from the taxonomy are shown in italics).

1) Quantitative Multistructural Level: Students were asked to compute a load flow and describe the operational issues that the system presented.

2) Qualitative Relational Level: Students were asked to analyze the condition of the system and to explain why this condition existed and why it was unacceptable. The students later applied their knowledge on power system analysis techniques to propose two designs.

3) Extended Abstract Level: The students were asked to hypothesize on possible design solutions and to test each of them. They were then asked to reflect on their designs, to judge which solution was best under the given design constraints, and to argue their choice based on their knowledge and previous analyses.

This approach incorporates several important elements that encourage deep learning, in that it does the following:

- sets criteria demanding learning at the highest cognitive levels, particularly at the extended abstract level [25];

- promotes a divergent ability [16], i.e., it generates alternatives that give the potential for other assessments of value such as originality, and creativity;

- promotes reflective learning, in that the guidelines and suggested tasks provided students with a series of questions triggering reflection;

- uses simulations that allow students to adapt parameters and make changes to answer "what would happen if. ..?" questions; 
- provides a context where unintended learning outcomes can flourish;

- promotes the use of FOSS, enabling the students to explore an unbounded set of possibilities and respecting their freedom to do so.

Some quotations given below, excerpted from student projects, suggest that the design of the project and the availability of a FOSS for power system analysis were enablers for the student learning and successful project completion. First, one of the student groups recognized the challenges in achieving high-level cognitive demands. Here, the FOSS PSAT acted as an enabling tool, allowing the group to reach the project goals.

The most difficult part of this project was understanding what was happening in the system and how to use our knowledge of power systems and the software to find a practical solution.

Moreover, it is important to recognize that this project provided a learning context that allowed unanticipated learning outcomes. The following quote is drawn from the conclusions of one of the projects.

Although we eliminated all of the system violations and corrected the bus voltages to within the desired 5\% deviation ${ }^{\alpha}$, another problem still remains. .. ${ }^{\beta}$ However, it would be extremely wise to construct several new transmission lines in order to maintain overall stability to the system. This would provide more reliable service to the system and eliminate the potential for a costly and perhaps catastrophic fault $\gamma$.

In this excerpt, it can be observed that at the point $\alpha$, the students had satisfied the design criteria and project requirements. They could have stopped at this point, but they had a "need to know" and, at the point $\beta$, identified additional issues with their design. As an unintended learning outcome, the students applied their knowledge on security and stability assessment by elaborating a third solution that also addressed these additional technical issues (at $\gamma$ above). This learning opportunity would have been unavailable if the course project had not encouraged the deep learning approach.

\section{Assessment and Grading for Functioning Knowledge}

Assessment involves a judgment of how well the student's design took into account the constraints and limits outlined in the project description. The important factor here is that the students must show an understanding of near-real-life limitations and how the problem can reasonably be attacked. This is why an open-ended assessment was chosen.

For the project assessment, three stages were used: 1) criteria for assessment were set; 2) the students selected the evidence to be used for judging their work against the criteria; 3 ) a judgment was made to determine if the criteria were satisfied by the selected evidence. The assessment format for this project was a group project, while the grading was performed using a set of high-level criteria (see Appendix B) instead of methods used in summative assessment that reward marks to each completed task. Evidence was selected by the students, and a reflective group report submitted by each of the student groups was read. A group grade was issued to the students. Although an individual grade could have been determined for each student based on their individual reported efforts, it was found that the students worked cooperatively during the whole project, and thus this was unnecessary. The reflective reports showed that they had applied the knowledge acquired in the course and that the deep learning approach was triggered as discussed in the previous section. Because of the value that the students attached to completing this project, there were no incidents requiring regrading and most students were satisfied with their grade, with most of the students ( 8 out of 10) deserving and being awarded the highest mark on the project.

Nevertheless, this learning activity and its assessment were not a determining factor for the final grade of the course. Because of this, it is likely that the students undertook both the surface approach (for the final examination particularly) and the deep approach (for the course project) as their strategy for dealing with the course.

The student interviews reported in Section V reveal that, despite this drawback, the course project using PSAT triggered the deep learning approach, which enabled long-term functioning knowledge. However, to exploit these benefits to the fullest extent, CA should be used for future course design, and the course TLAs, ILOs, and assessment should be in complete alignment.

\section{Triggering Deep Learning in Research Activities}

Since January 2003, when PSAT became available online, contributions in terms of new device models and algorithms have been very scarce. This is unusual for open-source projects, which typically take advantage of user contributions for quick development and extension of the original code. An analysis of the reasons for this failure of the PSAT as a FOSS can be found in [12].

The meager participation in the development of PSAT implies that while the community of PSAT users is relatively large, the community of PSAT developers is limited to a very few people, generally working in collaboration with the principal PSAT developer. Closely related to the clear difficulty in creating a community of PSAT developers is the difficulty of getting into the PSAT code. In fact, the current publicly available version of PSAT was not planned for cooperative development, but rather for being developed by one person. This is due to the fact that the main software architecture choices had already been before most of PSAT was made available.

Based on this experience, the second author of this paper has developed a new software package, largely from scratch, but with constant reference to at the legacy of PSAT. Experience both as a developer and a patent-holder of software architecture and as a supervisor of Ph.D. students was taken into account. The main objectives in developing this new project were to use the following:

1) an architecture flexible enough to cover any possible power system device model and solver algorithm;

2) a sufficiently modular structure to allow a concurrent utilization by the main developer as well as by a variety of other researchers and students; 
3) a hierarchical, class-based organization of device models that also allows a nonexpert user (e.g., undergraduate students working on their final project) to be able to write their own code that seamlessly integrates with the main project distribution.

After much reflection, it was clear that these goals could not be obtained by simply rewriting some parts of PSAT. The main issue was the language itself, i.e., MATLAB. Paradoxically, the most commonly used scientific-oriented computer language was actually a strong limitation to the deep learning of the PSAT architecture. This does not mean that the MATLAB language is not "good." It has undoubted merits, as shown by its use worldwide. However, the MATLAB language lacks, or makes it difficult to take advantage of, some basic features of modern object-oriented, high-level computer languages, such as dynamic typing, meta-programming, introspection, or polymorphism, all of which are beyond the scope of this paper. Further insights on computer language semantics can be found, for example, in [26].

A natural consequence of the limitations of MATLAB as a computer language is that MATLAB becomes intrinsically "difficult" to understand and to use for a complex software project. Again, the authors do not want to suggest that the MATLAB language cannot be used for complex projects - a huge variety of complex projects are based on MATLAB. Rather, MATLAB might not be a good choice if the goal is to develop a complex software project while simultaneously having to explain the project architecture to others, such as students who are not necessarily skilled in the computer programming techniques necessary for a complex software project.

In summary, if a project oriented to power system analysis is to be used as a springboard for research, such a project has to be easily understood. On the other hand, simplicity should not result in reducing or limiting the versatility and the generality of the project itself. It appears that an adequate solution, able to accommodate these two apparently incompatible requisites, is to organize the project as a layered and modular software architecture.

The top layer is the one that an end-user is going to use (i.e., the user interface). The second layer is composed of device models. Users interested in modifying or adding a new device only need to know the general device structure. In other words, the user is not forced to know low-level device functions, but only top-level ones. These can be easily obtained in classes and with meta-programming. A deeper layer is that implementing routines that handle devices, such as time-domain integration. A user who wants to define a new algorithm for time-domain integration has to not only understand the basic functioning of each device, but also how devices interact with the whole system. Writing a new algorithm requires a deeper understanding of the code, but is relatively less common than the need to implement a new device model.

Fig. 1 illustrates the organization of a power system software project in layers. The figure shows that there is still a need for a project developer who knows all the details of the project, as this is the common situation in most FOSS projects. It must be stressed that the layer approach is also an indicator of the level of the surface/deep learning of the user. As indicated in
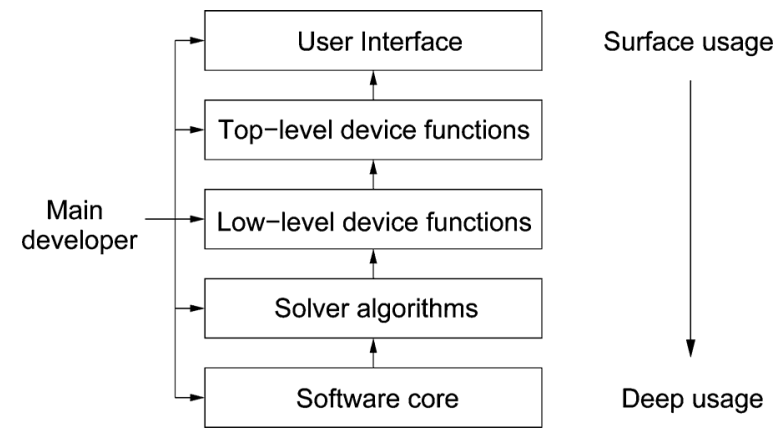

Deep usage

Fig. 1. Layer organization of a FOSS power system software project oriented to teaching and research.

Section II, these approaches are not mutually exclusive, but can always coexist. As a matter of fact, the same main developer is a surface user when running the program user interface.

The main issue with the MATLAB-based version of PSAT has been, and still is, that it mixes all the layers together, especially those dealing with device modeling. Clearly, for the main developer mixing everything together is not a problem, since she/he has in any case to be familiar with all the layers. However, if she/he needs to explain the code to others, the organization in layers plays a key role.

The need for meta-programming and a strong class-oriented programming led to the risky choice of abandoning MATLAB and adopting Python [13], [27]. The interested reader can find a very interesting discussion on the advantages of this language for general-purpose scientific programming in [28], while an extensive monograph on the organization and the results obtained with the new Python-based project can be found in [29].

The Python project was structured using a modular architecture so that new devices and new algorithms can be implemented as plug-ins. In this way, different people can work on different features of the program without interfering with each other. Moreover, the deep usage of Python class facilities helps to ease the students' learning process. Modularity and class-oriented programming have proved to be key factors for the success of a Python-based PSAT project.

In this paper, only the results of using the Python-based project for device development are reported. To this end, the experience of the second author in his supervision of the final project of an undergraduate student at the University of Castilla-La Mancha (UCLM), Ciudad Real, Spain, and his collaboration with two visiting Ph.D. students is discussed.

\section{A. Undergraduate Final Project}

The project offered to the undergraduate student consists of implementing a superconducting magnetic energy storage (SMES) device and its controllers. The objective was to implement an SMES control able to keep the output power produced by a wind park as constant as possible, regardless of wind speed variations. The overall scheme of the SMES and its controllers is shown in Fig. 2. By taking advantage of the modularity of the software tool, the student could reuse the wind turbine and the VSC device and its controllers (i.e., the regulators of the modulating amplitude $m$ and the firing angle $\alpha$ ), which had already been implemented, and focus on the SMES and the 


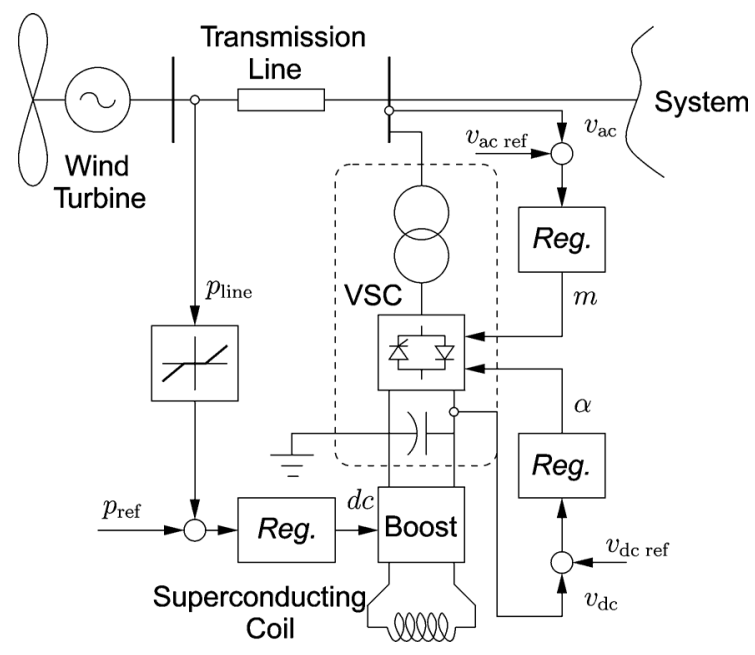

Fig. 2. Synoptic scheme of the SMES device, its controllers, and the coupling to a wind turbine.

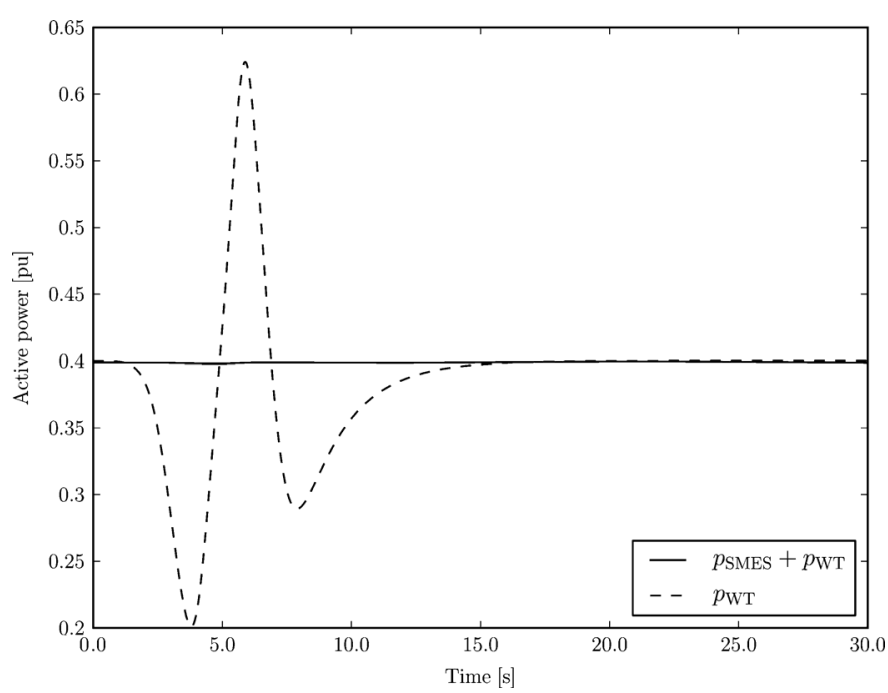

Fig. 3. Time-domain simulation illustrating the effect of the SMES when coupled to a wind turbine.

duty cycle $(D C)$ controller. Even though the student had no previous knowledge of the Python language, he was quickly able to master the code that he had to implement and was able to focus exclusively on modeling issues. For lack of space, the code implemented by the student cannot be shown here, but it is surprisingly simple and relatively short (about 150 lines).

A sample time domain simulation result of the project is shown in Fig. 3. The dashed line is the wind turbine active power output following a given perturbation, i.e., a Mexican-hat wind speed variation. The continuous line indicates the sum of the wind turbine active power output plus the SMES active power output. The simulation proves that, if properly tuned, the SMES controller is able to charge and discharge the superconducting coil in order to follow the assigned active power reference (in this case, $p_{\text {ref }}=0.4 \mathrm{pu}$ ). Other interesting results can be investigated - for instance, if the energy stored in the SMES is not sufficient to provide all needed active power, or if the coil reaches its maximum stored energy limit. How to handle limits has been one of the most relevant parts of the project.

\section{B. Ph.D. Student Activities}

The software was explained to two visiting Ph.D. students at UCLM. The visits were oriented to the development of new models of wind turbines (in particular, direct drive synchronous machines, DDSG) and photo-voltaic (PV) cells, respectively. The duration of the visit was limited to three months, so there was a need to reduce the initial software learning phase in order to begin the model implementation phase as soon as possible. The results were unexpectedly positive. Neither student knew Python before arriving at UCLM, but in just three weeks, both were able to start implementing the new models. At the end of the visiting periods, five DDSG, six PV cells models, and two sun irradiation models had been fully implemented and tested. In both cases, the devices implemented include tens of state and algebraic variables, complex nonlinearities involving variable hard limits, and intrinsic modeling complexity. Even though these students demonstrated quite exceptional skills and a clear deep learning attitude, these results would not have been possible using the MATLAB-based version of PSAT.

\section{STUdent InTERVIEWS}

\section{A. Approach and Design of the Student Interviews}

Three out of 10 students from the course "EPOW 4010-Power Engineering Fundamentals" taught during Fall 2006 at Rensselaer Polytechnic Institute, Troy, NY, were contacted to fill out the interview given in Appendix B. Performing these interviews three years after the students took the course, and now that they are part of the professional workforce, might be seen as unconventional. However, the authors believe that this approach allows it to be determined if deep learning was triggered, hence enabling long-term functioning knowledge.

Nevertheless, the drawback of this approach is that some of the students did not have a complete recollection of the details the authors were probing. It is recommended that those attempting a similar study carry out two sets of interviews, the first being immediately after the course has been completed. The first interview can be recorded, and transcripts saved to provide materials for analysis. A second interview after the students have graduated and are part of the active workforce (as here) can be most helpful in determining if functioning knowledge was achieved through learning activities that trigger the deep learning approach.

The questions from the interviews given in Appendix B have a heading in boldface indicating what was being probed. These headings were not distributed to the students, but are included here so that it is possible to correlate the critical analysis given. The questions focus only on the course project that used PSAT as its main tool, and not on the course as a whole, hence allowing analysis of the impact of this FOSS in their learning.

In contrast to the standard practice of using multiple-choice questions (MCQs), these student interviews were formulated using open-ended questions that required the ex-students to make written replies. The only drawback to this approach is 
that no statistical analysis can be performed, as when MCQs are used [11]. However, given the small course enrollment, in this case such statistics might be less meaningful than the analysis from student feedback.

\section{B. Analysis of Interviews}

Responses of three students to the questionnaire in Appendix B were compiled and analyzed. Selected excerpts of these answers and the authors' reflection are provided.

The students' overall impression of the project was quite positive. It was found that the approach used in the project design effectively employed the recommendations in [22], creating a route to understanding through application that prepared students for engineering practice. This is highlighted by the responses of the students to Q.1.

It was nice to work on a project similar to what you would be working on when in the workforce. While in school you tend to do a lot of smaller problems by hand, but in reality, you would be looking at a power system in whole and seeing the effects of the changes. It was good to replicate that with the project.

Another similar quote of a student's response to Q.1 was the following.

This was one of the more interesting projects that I had as an undergraduate, it had a clear goal but very openended ways to achieve that goal, giving us the freedom to simply experiment with the system and learn how a real power system would work.

The effect on functioning knowledge for professional practice of the project design approach should not be underestimated, as is made clear by a response to Q.2.

In the workforce everything can be open-ended. The customer is relying on your recommendation based on the study. So open-ended problems, although uncomfortable, are good for practical problems.

More importantly, an illuminating response to Q.18 highlights how the course project using PSAT allowed the students to engage in deep learning and how it enabled their long-term functioning knowledge.

My current job is basically a much larger-scale version of the course project, as we are examining deficiencies in the transmission system and trying to find cost-effective ways to cure these problems.

To support this evidence, an answer to Q.19 shows how, through the course project using PSAT, it was possible to lay a strong foundation of long-term functioning knowledge.

The project did a good job of showing the basic concepts of power engineering, which enabled me to start my job with a good knowledge of the conceptual workings of a power system. This has let me quickly expand these concepts to a larger power system, and get a good intuitive feel for how the power system will function with a number of different upgrades added to the system.
Having the FOSS PSAT allowed students to simplify their workflow, and the user friendliness (described in detail in [12]) is an enabling factor that other packages do not offer. This is reflected by the student when responding to Q.8.

Made things easy. When justifying a suggestion all you had to do was plug in the parameters, run the program, and explain the results.

A similar supporting answer is given by another student to Q.3.

It was much easier to use (as a beginner) than many other proprietary power flow programs I have used since, especially on a simple system (such as the one used in the project).

More importantly, while it might appear surprising to the reader, FOSS projects such as PSAT are usually of high quality compared to proprietary software. This is evidenced by an answer to Q.20.

The user interface was easy to learn and made it easy to quickly apply different solution ideas to the power system. Without this, text-based programs (including some of the proprietary programs which I use in industry today) make it much harder to quickly test and evaluate different solutions, and more difficult to see how power flows are distributed across the system.

To Q.23, asking if there should be changes to the project, one of the former students replied with the following answer.

No changes. Just add one or two more smaller exercises during the semester. The students will probably complain, but who cares what they think. They'll thank you later.

The questioned student is referring to "Preparatory Learning Activities" in Section III-A. It is interesting to note that the student states that even though students might "complain" about having more exercises in class, it will be beneficial in the long term. It is quite unusual that a former student asks for more learning activities, thus proving the positive feelings the learning activities generated.

\section{CONCLUSION AND Discussions}

This paper has shown that FOSS has great potential to support teaching and learning activities for undergraduate education, and research training at the graduate level. As shown in this paper, if used appropriately, FOSS can provide a useful tool for implementing CA theory at different university education levels. The use of PSAT aided in triggering the deep learning approach at the undergraduate level through class activities by allowing students to ask "what if?" questions by freely modifying the source code in the software, which would have been impossible without a FOSS. In addition, students were free to explore different alternatives for the designs in their project course, thanks to the features of PSAT as an educational FOSS.

A claim could be made for the possibility of achieving the same effect by using any proprietary tool with good visualization features, and for the value of exposing students to the propriety software they might use in industry. However, this 
claim cannot be sustained, from both a technical and ethical perspective. First, from the technical perspective, the preparatory learning activities could not have been carried out since these required changes to the source code; these activities are necessary so that the students can be successful in their project. Second, and more importantly, the question of value is merely a question focusing on narrow practical concerns [30]. There is a short-term economical value in having students become familiar with proprietary software used in industry, and thus reducing the cost of training for them to use the software. However, this short-term economic advantage comes at the cost of reducing the freedom of each individual user [30]. To be strict, this question should be addressed from an ethical perspective [30], [31]. The fact is that by obliging students to use proprietary software, the teacher becomes the accomplice of any software vendor who is restricting the freedom of the users of their software. FOSS is concerned with respecting the liberties of individual computer users [31]. This means that by using FOSS, the liberties of each individual student are guaranteed: It makes sure that students do not become prisoners of the software that they use.

Although the accomplishments reported in this paper are encouraging, the authors wish to raise a voice of concern: Despite the many efforts [10]-[12] made to enhance education through FOSS, it is expected that the massive adoption of FOSS for education in power engineering will continue to be a failure [29]. An educational change is required so that engineering students are taught to question commonly accepted assumptions and simplifications and are motivated to understand the intriguing mechanisms between theory, modeling, and scripting. This educational change will not take place if the appropriate tools for teaching are not embraced. Currently, education is carried out with restrictive proprietary software tools that do not allow students to explore beyond the limits of these tools. To add to that, the common surface learning approach is triggered by conventional teaching and assessment mechanisms that deprive the students of the possibility of awakening their "need to know."

The authors therefore hypothesize that this educational change can only be brought about through the implementation of teaching and learning methods from constructive alignment theory, where the deep learning approach is triggered through the use of FOSS, which provides students with the freedom to learn and explore - a freedom that is commonly denied by opaque proprietary software applications.

Finally, it can be noted that there is the need for FOSS projects specifically designed to be "good" deep learning triggers. The layer approach adopted for the next generation of Python-based PSAT projects appears to be a promising alternative. However, much work needs to be done in this particular area.

\section{APPENDIX A}

\section{Course ProjeCt DESCRIPTION}

Introduction: In this project, you will use your knowledge of electric power transmission, power engineering tools such as load flow analysis, and the PSAT to analyze the current status of the Modified IEEE 14 Bus test network and to propose solutions for normal operation of the network. Due to maintenance and insulation upgrading, some of the transmission lines of the system are out of service. Also, the system load has changed due to an increase of air conditioning loads. This results in the modified version of the IEEE 14 Bus system shown in Fig. 1 (not included here); you do not need to set up the system in PSAT, you will be provided with the PSAT-Simulink model for your convenience.

Design Objective: To propose and implement possible solutions to establish a normal operation of the system with a voltage deviation of $5 \%$ at all the buses, line loading below $100 \%$ in all the transmission lines, and no other limit violations in the network for all the scenarios of the study.

Suggested Project Tasks and Guidelines: The following tasks and guidelines will help you carrying out your project. While the only mandatory part to submit is Part III, the guidelines in Part I and Part II will give you a good framework to develop your project.

\section{Part I:}

1. Obtain the power flow solution for the initial conditions of the power system.

2. Analyze the line loading of each transmission line with the results obtained with the power flow solution. (To check if any line is violating its maximum capacity, click on the "Check limit violations" option before you produce a power flow report.)

3. Compute the total active and reactive power losses.

4. Obtain a voltage profile plot of the bus voltages.

5. Please describe the violations to the system operation.

Once the tasks described above have been carried out, answer the following questions making reference to the names or numbers of the buses or other elements of the power system:

a. Why is the current state of the system not acceptable for reliable operation?

b. From the power flow analysis: What abnormalities do you observe on the operation of the power system?

c. Regarding the former question: How do these abnormalities affect the satisfactory operation of the system?

d. What are your proposals to eradicate the existing abnormalities on the operation of the system? (Provide at least 2 alternatives)

e. Please make a technical-economical analysis of each of the proposals you have provided above. (This means you have to consider which of your options is the most economical while still satisfying the technical constraints making this the best choice. Note that you do not need to give actual real costs, you should rather do a qualitative comparison using your best engineering judgment.)

f. Select the most adequate and feasible option from the ones you have provided above and justify your choice from a technical-economical standpoint.

\section{Part II:}

For the alternative selected from your analysis of part one, please do the following:

1. Simulate the scenario of the alternative you have selected making all the changes necessary to the power network (adding lines, adding shunt capacitors, etc.).

2. Repeat all the numbered steps of part one (one to four). 
3. Provide comparative tables for the operating scenarios with and without the alternative you have implemented.

4. Do the system operating conditions improve? If they improve, how significant is the improvement? (You have to satisfy the design criteria of voltage deviation of $5 \%$ at all the buses, line loading below $100 \%$ at all the lines, and no other limit violations.)

5. Regarding the former step: Are the objectives of the project satisfied?

6. If the design criteria are not satisfied, you will have to select a new alternative to improve the operation of the system and repeat all of the above.

\section{Part III:}

This part is concerned with a report of your findings of Parts I and II. Your report must include the following elements; note that there is no template or required format, however the elements below should be present in some form in the report.

- A brief introduction summarizing the contents of your .

- A description of your design methodology.

- Present the technical-economical evaluations that led to your design (you must provide at least 2)

- Present your comparative results (from task 3 of part 2) (Graphs and Tables are strongly suggested.)

- A conclusion summarizing your findings and justifying your design from a technical-economical standpoint.

Note that presentation and neatness will be taken into account in your grade.

\section{Group Members}

You are required to make groups of at least two students and maximum three students and work as a cohesive unit for the development of the project. When you hand in your project, you must submit an additional page stating the contribution of each group member.

\section{Grading}

None of the tasks will be graded independently and no summative grade will be given for all of the individual tasks. Your project will be graded with the following criteria: i) evidence of the application of your power engineering knowledge; ii) the methodology used for your study; iii) the solutions you designed; and iv) your technical and economical arguments of the selected solution. An overall project grade will be given based on the grader's assessment of these criteria.

The project will be given an overall group grade that reflects the group effort to do the assignment. Additionally an individual grade will be given to each student for his personal contribution to the project.

\section{APPENDIX B}

\section{QUESTIONNAIRE FOR THE STUDENT INTERVIEWS}

\section{Teaching for Creativity}

1. Was there anything different in this course project to any other course project you carried out during your undergraduate studies?

2. This project had only two binding requirements, and provided a guide on how to perform the different analyses. Do you think having these kind of open-ended problems was positive or negative for your learning?

3. Thinking about what you had to do to complete the project, were the open-source features of PSAT something that facilitated you in performing the project?

4. Did the open-source nature of PSAT assist you in searching for the different options for your design?

5. Did PSAT gave you the freedom you needed to ask "what would happen if?" questions when you were doing your designs?

\section{Reflective Learning}

6. The project faced you with a power system with unacceptable operation performance and many challenges. While doing your different designs and selecting the final option, can you mention if you had any evaluation assessment to check your assumptions and the solutions you where proposing?

\section{Effect of Working in Groups}

7. You worked in your project with a partner. While coming up with different designs for the project, did the fact that you had a partner help you in coming up with different solutions?

8. Did using PSAT (i.e., having the same open tool) have an impact on working with your partner? Did it make things easy or difficult? Please explain.

\section{Intended Learning Outcomes}

9. The course teaches .... Do you think this course project helped you in achieving the objectives of the course?

10. Please explain if the use of PSAT for the course project aided in this matter?

\section{Teaching/learning activities for apply}

11. While doing your project, did you find yourself using the concepts of power flow given in class so to perform your analysis and designs?

12. Is there any particular example you can point to from the course project?

13. Do you think PSAT had any particular features that let you apply your knowledge effectively?

\section{Unintended Learning Outcomes}

14. Did you learn additional topics than those covered in the course through completing the course project?

15. Did using PSAT also help you in learning additional topics from other fields or developing other skills than those intended in the course covered in the course?

\section{Long-term Functioning Knowledge}

16. Where do you work now and what is your "title"?

17. What kind of activities do you carry out in your "title"?

18. Do you think the course project helped you in learning the topics that you need as a basis for your current job?

19. Have you found that the activities you had to perform in the project prepared you for dealing with more complex systems as those seen in your current work? 
20. Thinking about the way you used PSAT, is there any particular characteristic that enabled you to do your course project that you found useful?

21. Which features from the above would you like to use to perform power system analysis (or any other analysis and design) tasks in your current work?

\section{Additional Feedback}

22. Do you feel you got enough preparation in the use of PSAT to carry out the project? If not, was it easy or difficult to find your way around PSAT?

23. If this project were to be proposed again, what changes would you suggest to the description itself?

24. Did you get appropriate and timely feedback from the teaching assistant? What other things could the teaching assistant have done to help you?

\section{Comments}

Please add any additional feedback that you would like to give.

\section{ACKNOWLEDGMENT}

The authors would like to thank J. Crispino, D. Schwarting, and T. Fredeman for their feedback provided in the student interviews. The second author would also like to thank L. Calero, Dr. G. Forte, and Dr. A. Cagnano for being the first developers of the Python-based PSAT project and for their excellent work at UCLM.

\section{REFERENCES}

[1] B. Chowdhury, "Power education at the crossroads," IEEE Spectrum, vol. 37 , no. 10, pp. 64-69, Oct. 2000.

[2] G. Heydt and V. Vittal, "Feeding our profession (power engineering education)," IEEE Power Energy Mag., vol. 1, no. 1, pp. 38-45, Jan.-Feb. 2003.

[3] D. Niebur and D. Ray, "National Science Foundation Workshop on the Future Power Engineering Workforce," National Science Foundation, Arlington, VA, 2008 [Online]. Available: http://ecpe.ece.iastate.edu/ nsfws/

[4] "The EU's labour-shortage 'time bomb'," EurActiv.com, Brussels, Belgium, Jun. 2007 [Online]. Available: http://tinyurl.com/eu-shortage

[5] V. Gosbell and D. Robinson, E. Australia, E. S. A. of Australia, and E. E. S. of Australia, "Electrical power engineering: Assessing the future of electrical power engineering: A report on electrical power engineering manpower requirements in Australia," Engineers Australia, Barton, Australia, 2004.

[6] "Engineering: Turning ideas into reality-Fourth report of session 2008-09 House of Commons of the United Kingdom," The Innovation, Universities, Science \& Skills Committee, London, U.K., 2009 [Online]. Available: http://www.publications.parliament.uk

[7] "Serious shortage of engineers and specialists in the energy sector," Nordic Energy Research, Oslo, Norway, Jun. 2008 [Online]. Available: http://www.nordicenergy.org/onenews.cfm? Id=3-123\&path=

[8] M. J. Ashworth, "Preserving knowledge legacies: workforce aging, turnover and human resource issues in the US electric power industry," Int. J. Human Resource Manage, vol. 17, no. 9, pp. 1659-1688, 2006.

[9] F. Milano, "An open source power system analysis toolbox," IEEE Trans. Power Syst., vol. 20, no. 3, pp. 1199-1206, Aug. 2005.

[10] L. Vanfretti and F. Milano, "Application of the PSAT and open source software for educational and research purposes," in Proc. IEEE Power Energy Soc. Gen. Meeting, Jun. 2011, pp. 1-7.

[11] F. Milano, L. Vanfretti, and J. C. Morataya, "An open source power system virtual laboratory: The PSAT case and experience," IEEE Trans. Educ., vol. 51, no. 1, pp. 17-23, Feb. 2008.
[12] L. Vanfretti and F. Milano, "The experience of PSAT as a free and open source software for power system education and research," Int. J. Elect. Eng. Educ., vol. 27, no. 1, pp. 47-62, Jan. 2010.

[13] G. V. Rossum, "Python programming language," 2011 [Online]. Available: http://www.python.org

[14] G. Gentile, La Riforma dell'Educazione. Bari, Italy: Gius Laterza, 1920.

[15] G. Gentile, Il Concetto Moderno della Scienza e il Problema Universitario. Rome, Italy: Libreria di Cultura, 1921.

[16] J. Biggs and C. Tang, Teaching for Quality Learning at University, 3rd ed. Berkshire, England: Open Univ. Press, 2007.

[17] F. Marton and R. Säljö, "On qualitative differences in learning-I: Outcome and process," Brit. J. Educ. Psychol., vol. 46, no. 1, pp. 4-11, 1976.

[18] F. Marton and R. Säljö, "On qualitative differences in learning —i: outcome as a function of the learner's conception of the task," Brit. $J$. Educ. Psychol., vol. 46, no. 1, pp. 115-127, 1976.

[19] G. Gibbs, "Using assessment strategically to change the way students learn," in Assessment Matters in Higher Education: Choosing and Using Diverse Approaches. Berkshire, England: Open Univ. Press, 1999, pp. 41-53.

[20] K. Edström, J. Törnevik, M. Engström, and A. Wiklund, "Experiences of learning-Student accounts linked with theory," The Wallenberg CDIO Program, Royal Institute of Technology (KTH), Stockholm, Sweden, 2001 [Online]. Available: http://www.cdio.org/ files/exp_of_learning.pdf

[21] K. Edström, J. Törnevik, M. Engström, and A. Wiklund, "Studentens upplevelser av civilingenjörsutbildningen," Royal Institute of Technology (KTH), Stockholm, Sweden, 2003.

[22] K. Edström, J. Törnevik, M. Engström, and A. Wiklund, "Student involvement in principled change: Understanding the student experience," in Proc. 11th Improving Student Learning, Oxford, U.K., 2003.

[23] L. Vanfretti, "A brief introduction to the Power System Analysis Toolbox (PSAT) for power systems undergraduate courses," Nov. 2006 [Online]. Available: http://idisk.me.com/vanfretti/ Public/presentations/2006 LV BriefPSATTutorial 2006.pdf

[24] L. Vanfretti, "Power engineering fundamentals-Teaching and learning activities using PSAT," Nov. 2010 [Online]. Available: http:/ idisk.me.com/vanfretti/Public/pdfs/2010_LV_PEF_Compilation.pdf

[25] J. B. Biggs and K. F. Collis, Evaluating the Quality of Learning: The SOLO Taxonomy (Structure of the Observed Learning Outcome). New York: Academic, 1982.

[26] B. Pierce, Types and Programming Languages. Cambridge, MA: MIT Press, 2002.

[27] A. Martelli, Python in a Nutshell, 2nd ed. Sebastopol, CA: O’Reilly, 2006.

[28] H. P. Langtangen, Python Scripting for Computational Science, 3rd ed. Heidelberg, Germany: Springer-Verlag, 2002.

[29] F. Milano, Power System Modelling and Scripting. London, U.K.: Springer-Verlag, 2010.

[30] S. Chopra and S. D. Dexter, Decoding Liberation: The Promise of Free and Open Source Software. New York: Taylor \& Francis, 2008.

[31] R. M. Stallman, Free Software, Free Society: Selected Essays of Richard M. Stallman. Boston, MA: Free Software Foundation, 2002.

Luigi Vanfretti (M'10) received the M.S. and Ph.D. degrees in electric power engineering from Rensselaer Polytechnic Institute, Troy, NY, in 2007 and 2009, respectively.

He is an Assistant Professor with the Electric Power Systems Division, School of Electrical Engineering, KTH Royal Institute of Technology, Stockholm, Sweden. His research interests are modeling, dynamics, stability and control of power systems, applications of PMU data, and open-source software for power system engineering education.

Federico Milano (M'03-SM'09) received the Electrical Engineering degree and the Ph.D. degree in electrical engineering from the University of Genoa, Genoa, Italy, in 1999 and 2003, respectively.

From 2001 to 2002, he worked at the University of Waterloo, Waterloo, ON, Canada, as a Visiting Scholar. He is currently an Associate Professor with the University of Castilla-La Mancha, Ciudad Real, Spain. His research interests include voltage stability, electricity markets, and computer-based power system modeling and analysis. 